\title{
Chá: aspectos relacionados à qualidade e perspectivas
}

\author{
Tea: aspects related to the quality and prospects
}

\author{
Juliana Domingues Lima ${ }^{\text {I* }}$ Paulo Mazzafera ${ }^{\mathrm{II}}$ Wilson da Silva Moraes ${ }^{\mathrm{III}}$ \\ Reginaldo Barboza da Silva
}

- REVISÃO BIBLIOGRÁFICA -

\section{RESUMO}

O chá preto é uma das bebidas mais consumidas no mundo. Essa bebida é feita a partir da infusão de folhas processadas de Camellia sinensis, que é cultivada em mais de 30 países, tendo grande importância socioeconômica. Estudos sugerem que o chá tem efeito protetor contra diversos tipos de câncer e doenças cardiovasculares devido à presença de polifenóis denominados catequinas, que são oxidadas enzimaticamente durante o processamento das folhas, gerando uma mistura constituída principalmente de teaflavinas, teasinensinas e tearubiginas. A produção mundial de chá tem aumentado mais do que o consumo, provocando redução do preço que, juntamente com o aumento no custo de produção, implica a necessidade de alta produtividade e qualidade. Apesar disso, ainda não foram estabelecidos critérios precisos e eficientes para predizer a qualidade do chá a partir das folhas, bem como quais práticas agronômicas contribuem para o aumento da qualidade. No Brasil, a cultura do chá se concentra no Vale do Ribeira, em São Paulo (SP), sendo quase toda produção exportada. Apesar de o produto brasileiro não ser de alta qualidade, tem conseguido bons preços no mercado internacional. A produção brasileira, a área de produção e o número de indústrias de chá vêm diminuindo ao longo dos últimos anos, o que demonstra a necessidade de investimentos.

Palavras-chave: Camellia sinensis, catequinas, chá verde, chá preto, práticas agronômicas.

\section{ABSTRACT}

Black tea is one of the most widely consumed beverages in the world and it is prepared by the infusion of water with processed leaves of Camellia sinensis, which is cultivated in more than 30 countries, assuming a great socioeconomic importance. Several studies suggest that tea has protective effects against cancer and cardiovascular disease, due to the presence of polyphenols called catechins, which are oxidized enzymatically during the processing of the black tea, generating a mixture consisting mainly of theaflavins, theasinensins and thearubigins. The world production of tea has increased more than the demand, causing a drop of the price which, together with the increase in cultivation costs has shown a need for high productivity and quality. Nevertheless, there is not yet an efficient and accurate criteria to predict the quality of tea and agronomic practices which contribute to the increase in quality. In Brazil, the culture of tea is concentrated in the Ribeira Valley - SP, and almost all the production is exported. Despite the Brazilian product is not of high quality, it has achieved good prices in the international market. The Brazilian production, the production area and the number of tea industries are decreasing in recent years, clearly indicating the need for investments.

Key words: Camellia sinensins, catechins, green tea, black tea, agronomic practices.

ICampus Experimental de Registro, Universidade Estadual Paulista “Júlio de Mesquita Filho” (UNESP). Rua Nélson Brihi Badur, n. 430, Vila Tupy, 11900-000, Registro, SP, Brasil. E-mail: judlima@registro.unesp.br. *Autor para correspondência.

IIDepartamento de Fisiologia Vegetal, Instituto de Biologia, Universidade Estadual de Campinas (UNICAMP), Campinas, SP, Brasil.

IIIPólo Vale do Ribeira, Agência Paulista de Tecnologia dos Agronegócios (APTA), Pariquera-Açu, SP, Brasil. 


\section{INTRODUÇÃO}

Os chás produzidos a partir de folhas de Camellia sinensis L. O Kuntze. são classificados em três categorias conforme o processo de fabricação: fermentado (preto), não-fermentado (verde) e o semifermentado (oolong) (TANAKA\& KOUNO, 2003). No mundo, são produzidas anualmente cerca de três bilhões de toneladas de chá (KHAN \& MUKHTAR, 2007 ), sendo $78 \%$ preto, $20 \%$ verde e $2 \%$ semifermentado (KHAN \& MUKHTAR, 2007). Em conjunto, os três tipos constituem a bebida mais consumida no mundo (OWUOR et al., 2006; RUAN et al., 2007).

Estudos demonstraram que o chá verde tem efeito protetor contra diversos tipos de câncer e doenças cardiovasculares (YANG \& WANG, 1993; HIGDON \& FREI, 2003), possui propriedade antialérgica (SHIOKAZI et al., 1997), antiesclerótica (MIURA et al., 1994) e antibacteriana (HAMILTON-MILLER, 1995), além de ser rico em minerais e vitamina $K$ (MANFREDINI et al., 2004). O chá preto possui ação contra os radicais livres, tem potencial anticarcinogênico e antimutagênico (LIANG et al., 1999; CATTERALL et al., 2003) e capacidade para reduzir doenças cardiovasculares (DUFFY et al., 2001). Outros benefícios foram apontados em recente revisão elaborada por KHAN \& MUKHTAR (2007). Por essas razões, atualmente, o chá é considerado um alimento funcional que, se consumido no cotidiano, pode trazer inúmeros benefícios à saúde humana (KHAN \& MUKHTAR, 2007). Em contrapartida, alguns estudos evidenciaram efeitos danosos do chá à saúde, relacionando alguns de seus componentes orgânicos ou metais com neoplasias malignas (TAKAHASHI et al., 1992; SCHÜLLER et al., 2004), problemas renais (JACKSON \& HUANG, 1983), Mal de Alzheimer (WALTON et al., 1995), anemia (ROSEN, 1992) e fluorose dental (CAO et al., 1997).

C. sinensis é cultivada em mais de 30 países, tropicais e subtropicais (HAMPTON, 1992), sendo cultura de grande importância econômica e social. A produção mundial de chá tem aumentado mais do que seu consumo, causando redução no preço nas duas últimas décadas (ANON, 2001), além de aumento no custo de produção. Apesar de a atividade ainda ser viável economicamente e ser grande fonte de empregos (OWUOR \& OBANDA, 2007), a concorrência no mercado de chá tornou a qualidade o parâmetro mais importante para o estabelecimento do preço (RAVICHANDRAN \& PARTHIBAN, 1998). Esse cenário resulta na necessidade de aumento de rentabilidade por meio do aumento da qualidade do produto e da manutenção de altos índices de produtividade (OWUOR et al., 2006). Nesse sentido, esta revisão tem como objetivo destacar os aspectos agronômicos mais importantes para a definição da qualidade do chá e a situação atual do Brasil na produção mundial de chá.

\section{DESENVOLVIMENTO}

Composição química e tipos de chá

Polifenóis é um nome coletivo para compostos derivados do ácido cinâmico, dentre os quais, um dos grupos é denominado de flavonóides. Em C. Sinensis, esses flavonóides constituem cerca de 10-25\% da MS de folhas jovens e brotos, sendo denominados de catequinas (flavan-3-ols) (HAMPTON, 1992), classificadas nos subgrupos: catequina simples (C), epicatequina (EC), galatoepicatequina (ECG), epigalocatequina (EGC), galatoepigalocatequina (EGCG) e galocatequina-galato (GCG).

No chá verde, estão presentes, além das catequinas, outros compostos orgânicos, tais como cafeína e aminoácidos. A diferença entre o chá verde e o chá preto depende de quando as enzimas foliares são inativadas durante o processamento. Na fabricação do chá verde, as enzimas são inativadas imediatamente após a colheita das folhas (TANAKA \& KOUNO, 2003). Portanto, a composição de polifenóis no chá verde tende ser semelhante a das folhas frescas (TANAKA et al., 2003). Na produção do chá preto, as catequinas são oxidadas enzimaticamente, gerando uma mistura complexa de polifenóis, constituída de teaflavinas, teasinensinas e tearubiginas (TANAKA \& KOUNO, 2003), um exemplo específico do fenômeno conhecido como “escurecimento enzimático”, em que os compostos fenólicos são liberados no citoplasma quando o tecido é danificado por meio de técnica da fabricação (HASLAM, 2003).

Teaflavinas e teasinensinas são os principais produtos oxidados do chá preto (TANAKA et al., 2003), além das tearubiginas, presentes em menor quantidade. Teaflavinas dão coloração e brilho vermelho-laranja, sendo produzidas a partir da condensação oxidativa entre epicatequinas (EC) e epigalocatequinas (EGC). Teasinensinas são incolores, dímeros formados pelo acoplamento oxidativo da epigalocatequina (EGC) ou galato-epigalocatequina (EGCG) (HASHIMOTO et al., 1988), nos quais dois anéis $B$ de catequinas são conectados por meio de ligações covalentes do tipo C-C (TANAKA \& KOUNO, 2003). As tearubiginas possuem estruturas complexas e heterogêneas, monoméricas ou poliméricas, ainda não 
completamente elucidadas, com coloração marromferrugem, tendo como principais precursores a epigalocatequina e a galato-epigalocatequina (HASLAM, 2003).

Assim como no chá verde, o chá preto apresenta, na sua composição, além de polifenóis, outros compostos orgânicos como aminoácidos (1315\% da MS), metilxantinas (8-11\% da MS), carboidratos (15\% da MS), proteínas (1\% da MS), compostos voláteis ( $<0,1 \%$ da MS) e elementos minerais ( $10 \%$ da MS)(GRAHAM, 1992).

Uma vez que muitas das características sensoriais dos chás fabricados decorrem das transformações oxidativas dos fenóis da folha, grande atenção tem sido dada a esse tema. Anteriormente acreditava-se que o processo era fermentativo e, por esse motivo, ainda é conhecido como "fermentação", ao invés de oxidação (MATSUBARA \& RODRIGUEZAMAYA, 2006).

Dentre as enzimas envolvidas na oxidação, destaca-se a polifenoloxidase (PPO; EC 1.14.18.1.), localizada no cloroplasto (HASLAM, 2003), que, em uma das etapas da fabricação do chá preto, entra em contato com seus substratos armazenados no vacúolo (TAKEO, 1992). Ela atua promovendo a dimerização oxidativa de uma catequina simples (diidroxi) e uma galocatequina (triidroxi), gerando as diferentes teaflavinas: teaflavina simples (TF), teaflavina-3-galato (TF3g), teaflavina-3'-galato (TF3'g) e teaflavina-3-3'digalato (TF3,3'dg) (OWUOR et al., 2006). Outra enzima importante é a peroxidase [POD; EC 1.11.1.7], que catalisa a decomposição redutiva do peróxido de hidrogênio para a água, e peróxidos orgânicos ao seu correspondente álcool (HASLAM, 2003). Acoplada à POD, está a catalase (EC 1.11.1.16) (DIX et al., 1981), cuja função é remover rapidamente peróxidos gerados durante a oxidação das catequinas (NAKAYAMA et al., 2002).

Aspectos agronômicos e qualidade

A qualidade do chá verde é fortemente influenciada pelos componentes orgânicos e inorgânicos das folhas jovens e dos brotos, que funcionam como precursores e são alterados durante a sua transformação (aquecimento) em substâncias que determinam o sabor (HARA et al., 1995).

A cor, o sabor e o aroma do chá verde estão direta ou indiretamente associados às catequinas (WANG et al., 2000); portanto, são os principais compostos que definem a qualidade do chá verde. O sabor adstringente e amargo do chá verde é devido principalmente a esses polifenóis (RUAN et al., 2007). Aminoácidos livres são responsáveis pelo frescor e pela doçura da infusão do chá verde (WANG et al., 1988), além de contribuírem para formação de compostos voláteis responsáveis pelo aroma por reagirem com catequinas e açúcares solúveis durante o aquecimento (HARA \& KUBOTA, 1983). Dentre os alcalóides encontrados, destacam-se a cafeína por ser abundante (GRAHAM, 1992) e contribuir para o efeito estimulante e sabor (HARA et al., 1995), enquanto as clorofilas e a quercetina contribuem para coloração verde da infusão (WANG et al., 2004).

Para chá preto, apesar de o processo de oxidação ser considerado chave para definição da qualidade, os mesmos fatores que afetam a qualidade do chá verde também são importantes, como variação genética e condições ambientais e de cultivo (OWUOR \& OBANDA, 1995).

Para avaliação da qualidade do chá preto, são considerados o sabor, o aroma e a força de licor (BHATTACHARYYA et al., 2007), além dos atributos físicos e físico-químicos, tais como cor, textura, quantidade de resíduos, tipo e grau de enrolamento das folhas (OSAWA, 1990), teor de sólidos solúveis, viscosidade e densidade (BORSE et al., 2002). Até o momento, os chamados Tea Tasters, degustadores de chá, são os responsáveis por avaliarem os chás de maneira subjetiva (BHATTACHARYYA et al., 2007), pois não se conhece com precisão quais compostos químicos e características do licor mais se correlacionam com a qualidade do chá preto (OWUOR et al., 2006), nem foram desenvolvidos métodos para determinação da qualidade do chá (LIANG et al., 2003). Contudo, alguns componentes químicos têm sido considerados importantes, tais como as teaflavinas e as tearubiginas, que são responsáveis pela coloração, pelo brilho e pela força do licor (BISWAS et al., 1973). As diferentes teaflavinas conferem variados níveis de adstringência (SANDERSON et al., 1976); catequinas, cafeína e aminoácidos também são importantes para o sabor (SCHARBERT et al., 2004). Cafeína, além de contribuir para efeito estimulante, forma complexo com teaflavinas, que modifica o sabor, adiciona frescor à bebida e contribui para formação de precipitado colorido quando a infusão do chá preto é resfriada (OBANDA \& OWUOR, 1997).

Em países onde ainda há atrativos para expansão da produção de chá, a implantação ou reforma dos “chazais" deve ser feita com genótipos melhorados, com elevado potencial para qualidade do chá preto e não apenas de alta produtividade como tem sido feito até então (OBANDA \& OWUOR, 1997; OWUOR et al., 2006). Todavia, critérios precisos e eficientes para predizer a qualidade do chá preto a partir de folhas não foram estabelecidos (OWUOR \& OBANDA, 2007). 
A compreensão e o estabelecimento de parâmetros químicos nas folhas para predição da qualidade do chá, além de poderem ser utilizados em programas de melhoramento, poderiam servir para acompanhar mudanças nas práticas agronômicas ou na fabricação (OWUOR \& OBANDA, 2007). Já se sabe que os teores de polifenóis e catequinas e a atividade da polifenoloxidase nas folhas estão relacionados com a qualidade do chá preto (LOPEZ et al., 2005). Logo, uma questão importante a ser respondida é quais práticas agronômicas podem contribuir para o aumento da qualidade do chá e, conseqüentemente, da sua lucratividade (OWUOR \& OBANDA, 2007).

Devido ao fato de as diferenças na qualidade dos chás terem sido, muitas vezes, atribuídas à variação genética (OWUOR et al., 2006), o cultivo de chá tem sofrido uma transformação dramática em termos do tipo de muda utilizado para implantação de novos plantios (OWUOR \& OBANDA, 1999). Atualmente, as mudas em geral são propagadas vegetativamente a partir de clones, geralmente de híbridos selecionados com base na produtividade, qualidade ou resistência a condições ambientais adversas, ou ainda, na combinação dessas características (WACHIRA, 1994; SEUREI, 1996). Ocasionalmente ainda são utilizados genótipos de alta produção, mas de baixa qualidade ou vice-versa, que são processados isoladamente, e os produtos finais misturados. Contudo, clones de baixa qualidade têm dominância, reduzindo assim a qualidade do chá preto resultante (OWUOR \& OBANDA, 1999).

Também tem sido observada a influência das condições ambientais na qualidade do chá (McDOWELL et al., 1991). OWUOR (1992) verificou que variações na precipitação e temperatura afetaram o rendimento e a qualidade química e sensorial do chá preto do Kenya. Para o chá produzido na África Central, observou-se correlação positiva significativa entre teaflavinas totais e avaliação sensorial ou preço (HILTON \& ELLIS, 1972), mas essas correlações não foram significativas para o chá produzido no Kenya (OWUOR et al., 1986).

Nos diferentes países produtores, a produção de chá tem sido observada em diferentes altitudes. No entanto, o chá produzido em maiores altitudes e com temperaturas amenas apresenta crescimento mais lento e qualidade superior em termos de sabor e brilho, quando comparado com o chá cultivado em baixa altitude, com temperatura e umidade mais altas. Uma vez que não se sabe se o modelo de distribuição de teaflavinas é estável em diferentes condições ambientais, é preciso estudar o comportamento de um mesmo genótipo em diferentes regiões geográficas ou sob condições de cultivo contrastantes (OWUOR et al., 2006).
As características geoquímicas de rochas e solos são importantes fatores que afetam o crescimento de $\boldsymbol{C}$. sinensis e a qualidade do chá (GUOHUA et al., 1995), em parte pelo fato dessa espécie acumular metais essenciais ou tóxicos para maioria das plantas. Como conseqüência, pode haver acúmulo de metais benéficos ou prejudiciais à saúde humana na bebida de chá. Desse modo, o teor de metais tem sido considerado atributo de qualidade, sendo utilizado inclusive para inferir sobre o país de origem do chá (FERNÁNDEZ-CÁCERES et al., 2001). Dentre os metais acumulados, destaca-se o alumínio (Al), que pode ser acumulado em quantidades elevadas, superiores a $30 \mathrm{~g} \mathrm{~kg}^{-1} \mathrm{MS}$ (CHENERY, 1955), dependendo do genótipo (OWUOR et al., 1990a; SHU et al., 2003), sem sintoma aparente de toxidez.

Alguns trabalhos comprovaram que $\mathrm{Al}$ estimula o crescimento de $\boldsymbol{C}$. sinensis (KONISHI et al., 1985). Contudo, o modo pelo qual Al influencia o crescimento ainda não foi completamente elucidado. MARSCHNER (1995) atribuiu o efeito benéfico do Al ao alívio da toxidez causada por $\mathrm{H}^{+}$ou outro mineral, como, por exemplo, o fósforo. Segundo GHANATI et al. (2005), o Al induz aumento na atividade das enzimas antioxidantes, resultando no aumento da integridade da membrana, atraso na lignificação e envelhecimento da raiz. Para LIANG (1995), a tolerância ao Al pode ser atribuída à presença de ácidos orgânicos e polifenóis na raiz, que poderiam detoxificar esse metal por quelação. A quelação de $\mathrm{Al}$ por catequinas, outros polifenóis e ácidos orgânicos tem sido apontada como um modo eficiente de tolerância a esse metal (VITORELLO et al., 2005). Em chá, ocorre a quelação de Al por catequinas em folhas, uma exceção, pois normalmente esse metal não chega a ser transportado para a parte aérea da planta (VITORELO et al., 2005). $C$. sinensis também acumula flúor (F), que, por sua vez, pode complexar $\mathrm{Al}$, reduzindo sua fitotoxicidade (SHU et al., 2003). Considerando que $\mathrm{Al}$ pode interagir com catequinas, a qualidade do chá pode ser afetada pela presença desse metal.

Outros elementos minerais acumulados por C. sinensis são K e Mg (FERRARA et al., 2001), Mn, Ca, Zn, Cr e Ni (XIE et al., 1998), além de Se (ZHU et al., 2006) e F (SHU et al., 2003), sendo alguns destes essenciais para a saúde humana.

Tratos culturais têm sido citados como fatores que afetam a qualidade e os parâmetros de avaliação sensorial dos chás. A adubação com N e K, por exemplo, na razão 1:0,83, otimizou o sabor do chá e o teor de polifenóis totais e aminoácidos, em especial quando a dose de $\mathrm{N}$ foi 300 ou $450 \mathrm{~kg} \mathrm{ha}^{-1} \mathrm{ano}^{-1}$ (VENKATESAN \& GANAPATHY, 2004). RUAN et al. (2007) observaram que a adubação excessiva com N 
tem conseqüências negativas para o sabor do chá verde. $\mathrm{O}$ pH mais baixo do solo e o $\mathrm{N}$ na forma de $\mathrm{NH}_{4}^{+}$ favoreceram a formação de aminoácidos, theanina, polifenóis e cafeína. O aumento das doses e a freqüência da adubação nitrogenada e a aplicação de $\mathrm{N}$ adicionado de $\mathrm{P}$ e K reduziram o conteúdo de $\mathrm{Al}$ nas folhas (OWUOR et al., 1990a).

O controle de doenças é essencial, pois algumas ocorrem diretamente nas folhas novas e nos brotos do chá, podendo afetar tanto a produtividade, como a qualidade, sendo a deterioração da qualidade diretamente proporcional à severidade da doença (GULATI et al., 1999).

A qualidade química varia também em função da poda, que é uma prática essencial para obtenção de folhas novas, matéria-prima para produção do chá (RAVICHANDRAN, 2004). Ela induz a ramificação da planta e, conseqüentemente, a produção de um maior número de folhas novas, uniformiza as faces para colheita mecânica das folhas (OSAWA, 1990) e promove a limpeza da planta e a remoção de ramificações velhas com vigor baixo.

Os precursores responsáveis pela qualidade do chá, tais como polifenóis, aumentaram seu conteúdo no primeiro ano após a poda e depois diminuiram com o aumento do tempo de poda (RAVICHANDRAN, 2004; THOMAS et al., 2005), já tendo sido comprovado que a qualidade do chá diminui com o aumento da taxa de crescimento da planta (OWUOR et al., 1990b).

O método de colheita também afeta a qualidade do chá produzido. Em estudo realizado por RAVICHANDRAN \& PARTHIBAN (1998), folhas colhidas manualmente foram as que apresentaram maiores teores de precursores bioquímicos importantes para a qualidade do chá preto em relação à colheita mecânica, que é não-seletiva e pode causar lesão por cisalhamento. No entanto, com o aperfeiçoamento tecnológico, o chá colhido mecanicamente freqüentemente tem apresentado qualidade similar ou superior ao chá colhido manualmente.

O ponto de colheita está relacionado diretamente com a qualidade do produto final, pois o grau de maturidade fisiológica das folhas afeta o conteúdo de catequinas (THOMAS et al., 2005). As brotações são classificadas antes do processamento industrial em diferentes tipos, com base no número e tamanho de folhas. Brotações de melhor qualidade são aquelas que apresentam apenas a gema apical com, no máximo, duas folhas novas, não completamente expandidas (OSAWA, 1990).
Situação atual do Brasil na produção do chá e perspectivas

No Brasil, a cultura do chá foi introduzida no Rio de Janeiro por D. João VI e espalhou-se para Minas Gerais, São Paulo e Paraná (OSAWA, 1990). Atualmente, a principal região produtora do chá no Brasil é o Vale do Ribeira, em São Paulo (SP), principalmente os municípios de Registro, PariqueraAçu e Cajati, sendo a colonização japonesa a grande responsável pela expansão dessa cultura na região.

Cerca de $99 \%$ do chá preto exportado pelo Brasil, em 2006, foi produzido no Estado de São Paulo (PÉREZ, 2007), sendo 95\% da produção paulista destinada a mercados externos, que utiliza o produto na composição de ligas em empresas como a Lipton/ Pepsi-Cola e Nestlé/Coca-Cola (VEGRO e BEMELMANS, 1996). Os principais compradores do chá preto são os Estados Unidos (37,7\%), seguido do Reino Unido (24,6\%), Chile (16,7\%) e Uruguai $(4,4 \%)$ (PÉREZ, 2007).

Na safra 2006/2007, a produção de chá preto no Vale do Ribeira, representada por quatro empresas, foi de 3.225ton, uma produção considerada baixa, quando comparada com a de 8.178ton obtida em 1993/ 1994 pela reunião da produção de sete empresas ativas. No período 1983-1994, a área colhida foi de 5.376ha, enquanto que no período de 1995-2002 foi de 3.701ha, segundo a Coordenadoria de Assistência Técnica Integral (CATI) e o Instituto de Economia Agrícola (IEA), ambos de São Paulo. Os dados mostram redução da atividade, apesar das exportações de chá terem gerado boas cifras para região, como em 2002, da ordem de 4,4 milhões de reais, o que representou 0,71\% do PIB daquele ano, segundo o Instituto Brasileiro de Geografia e Estatística (IBGE).

O chá preto produzido no Vale do Ribeira vinha sendo classificado como de baixa qualidade e vendido para formação de blend (mistura). Atualmente, com o plantio de genótipos melhorados, normalmente híbridos resultantes de cruzamentos do chá Chinês (o de folha estreita) e do chá Assam (o de folha larga), os tratos culturais e o processo de fermentação mais adequados, tem sido possível obter um produto de qualidade superior, especialmente pela sua cor, favorecendo a classificação e, como conseqüência, o preço. Isso tem permitido a inserção do produto brasileiro no mercado exportador mesmo com as dificuldades econômicas enfrentadas nos últimos anos.

\section{CONSIDERAÇÕES FINAIS}

Tendo em vista 1) a importância dessa cultura no cenário brasileiro e mundial; 2) as múltiplas 
possibilidades de comercialização dos produtos do chá verde ou preto para as indústrias alimentícia, de cosméticos e farmacêutica; 3) a localização geográfica onde a cultura se concentra no Brasil, o Vale do Ribeira, umas das regiões mais pobres do Estado de São Paulo; e 4) os poucos avanços da pesquisa brasileira para essa cultura; é evidente a necessidade de tomada de medidas no sentido de impedir que essa cultura seja negligenciada ou até extinta no Brasil, pois o momento é de grande pessimismo por parte dos produtores e empresários do setor. Assim, faz-se necessário um amplo levantamento dos problemas da cultura nos seus locais de produção, assim como a realização de pesquisas visando não só o incentivo para a expansão da cultura, como também a exploração do máximo potencial produtivo e a produção de chá de melhor qualidade, a exemplo do que tem sido feito para outras culturas no Brasil.

\section{AGRADECIMENTOS}

Os autores agradecem à Fundação para o Desenvolvimento da UNESP (FUNDUNESP), pelo auxílio financeiro para publicação e às empresas CHABRAS e YAMATEA, pelo fornecimento dos dados de produção de chá.

\section{REFERÊNCIAS}

ANON. International Tea Committee. London: Annual Bulletin of Statistics, 2001. 182p.

BHATTACHARYYA, N. et al. Detection of optimum fermentation time for black tea manufacturing using electronic nose. Sensors and Actuators B, Lausanne, v.122, n.2, p.627634, 2007. Disponível em: http://www.sciencedirect.com/ science?_ob=ArticleURL\&_udi=B6THH-4KSD5BB$1 \&$ \&user $=687358 \&$ \&doc $=1 \&$ \& $\mathrm{mt}=\&$ \&orig $=$ search $\&$ _sort $=$ d\&view $=$ c\&_acct $=$ C000037 899\&_version $=1 \&$ \&urlVer sion=0\&_userid=687358\&md5=3ae0b6dd19d3098ee5ba6a30c0373cae. Doi: $10.1016 /$ j.snb.2006.07.013.

BISWAS, A.K. et al. Biological and chemical factors affecting the valuations of North East India planters. III. Statistical evaluation of the biochemical constituents and their effects on colour, brightness, and strength of black teas. Journal of Food, Agriculture and Environment, Helsinki, v.24, p.14571477, 1973.

BORSE, B.B. et al. Fingerprint of black teas from India: identification of the region-specific characteristics. Food Chemistry, London, v.79, n.4, p.419-424, 2002.

CAO, J. et al. Brick tea consumption as the cause of dental fluorosis among children from Mongol, Kazak and Yugu populations in China. Food and Chemical Toxicology, Oxford, v.35, n.8, p.827-833, 1997. Disponível em: http:// w w w. s c i e n c e d i r e c t. c o m / science?_ob=ArticleURL\&_udi=B6T6P-3RJR062B\&_user $=687358 \&$ \&doc $=1 \&$ \&_fmt $=\&$ \&_orig $=$ search \&_sort $=$ d \& vi e w = c \&_a c c t = C $000037899 \&$ \& e r s i o n $=1 \&$ \& u r l V e r s i o $n=0 \&$ _ u s e r i d $=687358 \&$ md $5=2541433431311520 c 4 b 4 b 5 e 31956265 c$. Doi: 10.1016/S0278-6915(97)00049-5.

CATTERALL, F. et al. Effects of black tea theaflavins on aflatoxin $B_{1}$ mutagenesis in the Ames test. Mutagenesis, Oxford, v.18, n.2, p.145-150, 2003.

CHENERY, E.M. A preliminary study of aluminium and the tea bush. Plant and Soil, The Hague, v.6, n.2, p.174-200, 1955. Disponível em: http://www.springerlink.com/content/ u474pm2653675282/. Doi: 10.1007/BF01343446.

DIX, M.A. et al. Fermentation of tea in aqueous suspension: influence of tea peroxidase. Journal of Food, Agriculture and Environment, Helsinki, v.32, n.4, p.920-932, 1981.

DUFFY, S.J. et al. Short and long-term black tea consumption reverses endothelial dysfunction in patients with coronary artery disease. Circulation, Dallas, v.104, n.2, p.151-156, 2001.

FERNÁNDEZ-CÁCERES, P.L. et al. Differentiation of tea (Camellia sinensis) varieties and their geographical origin according to their metal content. Journal of Agricultural and Food Chemistry, Eanston, v.49, n.10, p.4775-4779, 2001. Disponível em: http://pubs.acs.org/doi/abs/10.1021/ jf0106143. Doi: 10.1021/jf0106143.

FERRARA, L. et al. The distribution of minerals and flavonoids in the tea plant (Camellia sinensis). II Farmaco, Pavia, v.56, n.5-7, p.397-401, 2001. Disponível em: http:// www.sciencedirect.com/science?_ob=ArticleURL\&_udi=B6VJ8$43 \mathrm{C} 5 \mathrm{D} 2 \mathrm{R}-\mathrm{F} \&$ \&user $=687358 \&$ \&_rdoc $=1 \&$ \& fmt $=\&$ \& orig $=$ searc h\&_sort $=\mathrm{d} \&$ view $=\mathrm{c} \&$ \&acct $=$ \&_version $=1$ \&_urlVersion $=0 \&$ \&userid=687358\&md5=9aa24b899776daf881757d8b0bc5a5d1. Doi: 10.1016/S0014-827X(01)01104-1.

GHANATI, F. et al. Effects of aluminum on the growth of tea plant and activation of antioxidant system. Plant and Soil, The Hague, v.276, n.1-2, p.133-141, 2005. Disponível em: http://www.springerlink.com/content/y22642163h17r733/. Doi: 10.1007/s11104-005-3697-y.

GRAHAM, H.N. Green tea composition, consumption, and polyphenol chemistry. Preventive Medicine, San Diego, v.21, n.3, p.334-350, 1992. Disponível em: http:// w w w. s c i e n c e d i r e c t.c c m / science?_ob=ArticleURL\&_udi=B6WPG-4BSVKPG$1 \mathrm{R} \&$ \& u s e r $=687358 \&_{\text {_ }} \mathrm{rd} \mathrm{o} \mathrm{c}=1 \&_{\text {_f }} \mathrm{m} \mathrm{t}=\&_{-} \mathrm{o}$ rig = s e a r c h \& s o r t $=$ d \& vi e w $=$ c \& _ a c c t $=\mathrm{C}$

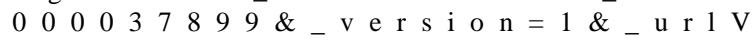
ersion=0\&_userid=687358\&md5=287cd0b73eb34a9abc422dd0abb95d12. Doi: $10.1016 / 0091-7435(92) 90041-F$.

GULATI, A. et al. Variation in chemical composition and quality of tea (Camellia sinensis) with increasing blister blith (Exobasidium vexans) severity. Mycological Research, Cambridge, v.103, n.11, p.1380-1384, 1999.

GUOHUA, Z. et al. Geochemical characteristics affecting the cultivation and quality of Longjing Tea. Journal of Geochemical Exploration, Amsterdam, v.55, n.1-3, p.183191, 1995.

HAMILTON-MILLER, J.M. Antimicrobial properties of tea (Camellia sinensis L.). Antimicrobial and Agents Chemotherapy, Washington, v.39, n.11, p.2375- 2377, 1995. 
HAMPTON, M.E. Production of black tea. In: WILLSON, K.C.; CLIFFORD, M.N. (Eds.). Tea cultivation to consumption. London: Chapman \& Hall, 1992. p.459-510.

HARA, T.; KUBOTA, E. Volatile compounds formed on roasting catechine with L-theanine. Study of Tea, Tokyo, v.64, p.3233, 1983.

HARA, Y. et al. Chemical composition of tea. Food Reviews International, New York, v.11, p.435-456, 1995.

HASLAM, E. Thoughts on thearubigins. Phytochemistry, New York, v.64, n.1, p.61-73, 2003. Disponível em: http:// w w w . s c i e n c e d i r e c t. c o m / science?_ob=ArticleURL\&_udi=B6TH7-49C4FXG$4 \&$ \&user $=687358 \&$ \&doc $=1 \&_{-}$fmt $=\&_{-}$orig $=$search $\&_{-}$s or t $=$d \& vi e w $=$c \& $\&_{-}$a c c t $=$C 0000037 899 \& v e r s i o $n=1$ \& u r l V e r s ion $=0 \& \_$userid $=687358 \& \mathrm{md} 5=5 f 55969087 \mathrm{c} 40 \mathrm{e} 09 \mathrm{afb} 2 \mathrm{c} 724 \mathrm{~d} 0 \mathrm{bc} 8 \mathrm{ecf}$. Doi: 10.1016/S0031-9422(03)00355-8.

HASHIMOTO, F. et al. Tannins and related compounds. LXIX. Isolation and structure elucidation of B,B'-linked bisflavanoids, theasinensins D-G and oolongtheanin from oolong tea. Chemical \& Pharmaceutical Bulletin, Tokyo, v.2, n.36, p.1676-1684, 1988.

HIGDON, J.V.; FREI, B. Tea catechins and polyphenols: health effects, metabolism, and antioxidant functions. Critical Reviews in Food Science and Nutrition, Boca Raton, v.43, n.1, p.89-143, 2003. Disponível em: http:// w w w. i n f o r m a w o r l d c c o m / s m p p / content $\sim \mathrm{db}=$ all ? content $=10.1080 / 10408690390826464$. Doi: $10.1080 / 10408690390826464$.

HILTON, P.J.; ELLIS, R.T. Estimation of the market value of Central African tea by theaflavins analysis. Journal of the Science of Food and Agriculture, London, v.23, n.2, p.227232, 1972.

JACKSON, M.L.; HUANG, P.M. Aluminum of acid soils in the food chain and senility. Science of the Total Environment, Amsterdam, v.28, n.1-3, p.269-276, 1983. Disponível em: h t t p : / / w w w. s c i e n c e d i r e c t. c o m/ science? ob $=$ ArticleURL\&_udi=B6V78-4HHXWYNV\&_user $=687358 \&$ \& rdoc $=1 \&$ \&_fmt $=\&$ \&orig $=$ search \&_s o r t $=$ d \& vi e w = c \& a c c t $=$ C 000003789 9 \& _ v e r s i o n $=1$ \& _ u r l V e r s i o $n=$ 0\&_userid=687358\&md5=89ffc805d17aabf7ff75860dd0f85516. Doi: 10.1016/S0048-9697(83)80024-2.

KHAN, N.; MUKHTAR, H. Tea polyphenols for health promotion. Life Sciences, Oxford, v.81, n.7, p.519-533, 2007. Disponível em: http://www.sciencedirect.com/ science?_ob=ArticleURL\&_udi=B6T99-4P2S983-



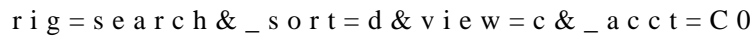
$\begin{array}{llllllllll}0 & 0 & 0 & 3 & 7 & 8 & 9 & 9 & \&\end{array}$ IVersion=0\&_userid=687358\&md5=76b1f32797d23d593f4b21c6a8173ca8. Doi: $10.1016 / j .1 f s .2007 .06 .011$

KONISHI, S. et al. Stimulatory effects of aluminum on tea plants growth under low and high phosphorus supply. Soil Science and Plant Nutrition, Tokyo, v.31, p. 361-368, 1985.

LIANG, J.Y. Avoidance mechanisms of nutritionalphysiological interference of aluminum in the tea plant
(Camellia sinensis). 1995. 155f. Thesis (Ph.D. in Agronomy) - National Taiwan University, Taipei, Taiwan.

LIANG, Y.C. et al. Suppression of extracellular signals and cell proliferation by the black tea polyphenol, theaflavin-3,3'digallate. Carcinogenesis, Oxford, v.20, n.4, p.733-736, 1999.

LIANG, Y. et al. Estimation of black tea quality by analysis of chemical composition and colour difference of tea infusions. Food Chemistry, London, v.80, n.2, p.283-290, 2003. Disponível em: http://www.sciencedirect.com/ science?_ob=ArticleURL\&_udi=B6T6R-47CDXP1$3 \&$ \&user $=687358 \&$ \&doc $=1 \&$ \& fmt $=\&$ _orig $=$ search $\&$ sort $=$ d \& vie w $=$ c \&_a c ct $=$ C $000037899 \&$ _ ve r s i o n $=1$ \& _ u r l Version $=0$ \& _ us e rid $=687358 \& m d 5=79 a a 6 c e 494 b f 80 a a 726 f c 0 f e 7 c f f e b 72$. Doi: 10.1016/S0308-8146(02)00415-6.

LOPEZ, S.J. et al. A reliable technique to identify superior quality clones from tea germplasm. Food Chemistry, London, v.91, n.4, p.771-778, 2005. Disponível em: http:// w w w. s c i e n c e d i r e c t. c o m / science?_ob=ArticleURL\&_udi=B6T6R-4F14YV8$2 \&$ _user $=687358 \&$ \&rdoc $=1 \&$ fmt $=$ \&_orig $=$ search\&_sort $=\mathrm{d}$ $\&$ view $=c \&$ acct $=$ C $000037899 \&$ version $=1 \&$ \& urlVe rsion=0\&_userid=687358\&md5=9477c7a51d91ac0063e43882f11c9880. Doi: 10.1016/j.foodchem.2004.10.005.

McDOWELL, I. et al. Phenolic compounds of black tea liquors as a means of predicting price and country of origin. Journal of the Science of Food and Agriculture, London, v.55, n.4, p.627-641, 1991.

MANFREDINI, V. et al. Chá verde: benefícios para a saúde humana. Infarma, Brasília, v.16, n.9-10, p.68-70, 2004

MARSCHNER, H. Mineral nutrition of higher plants. London: Academic, 1995. 889p.

MATSUBARA, S.; RODRIGUEZ-AMAYA, D.B. Teores de catequinas e teaflavinas em chás comercializados no Brasil. Ciência e Tecnologia de Alimentos, Campinas, v.26, n.2, p.401-407, 2006. Disponível em: http:// www.scielo.br/scielo.php?script=sci_arttext\&pid=S0101$20612006000200024 \& \operatorname{lng}=$ en $\& n r m=i s o \& t \operatorname{lng}=$ pt. Doi: 10.1590/S0101-20612006000200024.

MIURA, S. et al. The inhibitory effects of tea polyphenols (flavan-3-ol derivates) on $\mathrm{Cu}^{2+}$ mediated oxidative modification of low-density lipoprotein. Biological \& Pharmaceutical Bulletin, Tokyo, v.17, n.12, p.1567-1572, 1994.

NAKAYAMA, T. et al. Mechanisms and structural specificity of hydrogen peroxide formation during oxidation of catechins. Food Science and Technology Research, London, v.8, n.3, p.261-267, 2002. Disponível em: http://www.jstage.jst.go.jp/ article/fstr/8/3/8_261/_article. Doi: 10.3136/fstr.8.261.

OBANDA, M.; OWUOR, P.O. Flavanol composition and caffeine content of green leaf as quality potential indicators of Kenyan black teas. Journal of the Science of Food and Agriculture, London, v.74, n.2, p.209-215, 1997. Disponível em: http:// www 3.interscience.wiley.com/journal/ 6684 / abstract?CRETRY $=1 \&$ SRETRY $=0$. Doi: 10.1002/(SICI)10970010(199706)74:2<209::AID-JSFA789>3.0.CO;2-4. 
OSAWA, J.L. O chá do município de Registro e seus reflexos na expansão sócio econômica da região. Curitiba: Universidade Federal do Paraná, 1990. 75p.

OWUOR, P.O. et al. Correlation of theaflavins content and valuations of Kenyan black teas. Journal of the Science of Food and Agriculture, London, v.37, n.5, p.507-513, 1986.

OWUOR, O.P. et al. Levels of aluminium in green leaf of clonal teas, black tea and black tea liquors, and effects of rates of nitrogen fertilizers on the aluminium black tea contents. Food Chemistry, London, v.35, n.1, p.59-68, 1990a. Disponível em: http:// www.sciencedirect.com/science?_ob=ArticleURL\&_udi=B6T6R49 NR0WF- 1 XH\& user $=687358 \&$ rdoc $=1 \& \mathrm{fmt}=\&$ orig $=$ s e a r c h \&_s or t $=$ d \& vi e w $=$ c \&_a c c t $=$ C 0000 37899\&_version $=1 \&$ \&urlVersio $n=0 \&$ \&userid $=687358 \& \mathrm{md}$ 5=d8fc8912f42ece8c9ba685a62eadced9. Doi: 10.1016/03088146(90)90131-M.

OWUOR, P.O. et al. Effects of altitude on chemical composition of black tea. Journal of the Science of Food and Agriculture, London, v.50, n.1, p.9-17, 1990b.

OWUOR, P.O. Changes in quality parameters of commercial black seedling tea due to the time of the year in the Eastern Highlands of Kenya. Food Chemistry, London, v.45, n.2, p.119-124, 1992. Disponível em: http://www.sciencedirect.com/ science?_ob=ArticleURL\&_udi=B6T6R-49NPW085T\&_user $=687358 \&$ \&doc $=1 \&$ \& $\mathrm{mt}=\&$ \&orig $=$ search \& _ sort $=$ d \& vi e w $=$ c $\&$ _ a c c t $=$ C $000037899 \&$ \& ver s i o $n=1 \&$ \& r l Vers i o $n=0 \& \&_{\text {u s e r i d }=6}$ 87358\&md5=797ebff381679605cb02378500a4f6ce. Doi: 10.1016/0308-8146(92)90021-S.

OWUOR, P.O.; OBANDA, M. Clonal variations in the individual theaflavin levels and their impact on astringency and sensory evaluations. Food Chemistry, London, v.54, n.3, p.273-277, 1995. Disponível em: http://www.sciencedirect.com/ science?_ob=ArticleURL\&_udi=B6T6R-3YYT6DFS\&_use r $=687358 \&$ \&_d oc $=1 \&$ \&_fmt $=\&$ \&_orig $=$ sea r c h \& _ s o r t $=$ d \& v i e w $=$ c \& _ a c c t $=$ C 00000 $37899 \&$ \& version $=1 \&$ \&urlVersion $=0$ \&_userid $=687358 \& \mathrm{md} 5=\mathrm{f}$ fbf0dc38dbeeec7023ac28e86c64686. Doi: 10.1016/03088146(95)00046-L.

OWUOR, P.O.; OBANDA, M. The effects of blending clonal leaf on black tea quality. Food Chemistry, London, v.66, n.2, p.147-152, 1999. Disponível em: http://www.sciencedirect.com/ science?_ob=ArticleURL\&_udi=B6T6R-3WFN9M1$2 \&$ \&_user $=687358 \&$ \& rdoc $=1 \&$ \&_fmt $=\&$ \&orig $=$ se a r ch $\& \quad$ s o r t $=$ d \& vi e w $=$ c \& a c c t $=$ C 0000037899 \&__ v r s i o $n=1 \&$ _ u r l Ver s i o $n=0 \&$ _ u serid=687358\&md5=07a6aa2920acba082a06324c938daeb4 Doi: 10.1016/S0308-8146(98)00120-4.

OWUOR, P.O.; OBANDA, M. The use of green tea (Camellia sinensis) leaf flavan-3-ol composition in predicting plain black tea quality potential. Food Chemistry, London, v.100, n.3, p.873-884, 2007. Disponível em: http://www.sciencedirect.com/ science?_ob=ArticleURL\&_udi=B6T6R-4HYV0BT-

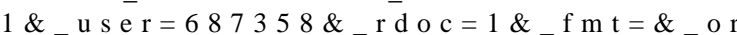
i g = s e a r c h \&_s ort $=$ d \& vi e w $=$ c \&_a c c t $=$ C 00 $0037899 \&$ \& version $=1 \&$ \& url Version $=$ 0\&_userid=687358\&md5=a0fc8f07a511f37c3fe061bfc0dbbef5. Doi: 10.1016/j.foodchem.2005.10.030.
OWUOR, P.O. et al. The relationship between some chemical parameters and sensory evaluations for plain black tea (Camellia sinensis) produced in Kenya and comparison with similar teas from Malawi and South Africa. Food Chemistry, London, v.97, n.4, p.644-653, 2006. Disponível em: http:// www.sciencedirect.com/science? ob=ArticleURL\&_udi=B6T6R4 GRH 7 C B $-1 \&$ __user $=687358 \&$ \&_rdoc $=1 \&$ \& f t $=\&$ orig $=$ s e a r c h $\&$ s ort $=$ d \& vi e w $=$ c $\&$ _ a c c t $=$ C $000037899 \&$ _ version $=1 \&_{\text {_ u r l Versio }}$ $\mathrm{n}=0$ \&_userid=687358\&md5=f2d22f765e0da2a5c7ebe1e050b71f2e. Doi: 10.1016/j.foodchem.2005.04.027.

PÉREZ, L. Chá, mate e especiarias: exportações brasileiras 1996 a 2006. Informações Econômicas, São Paulo, v.37, n.7, p.40-49, 2007.

RAVICHANDRAN, R. The impact of pruning and time from pruning on quality and aroma constituents of black tea. Food Chemistry, London, v.84, n.1, p.7-11, 2004. Disponível em: http:/ /www.sciencedirect.com/science?_ob=ArticleURL\&_udi=B6T6R48NBYK6-3\&_user=687358\&_rdoc $=18$ \&fmt $=$ \&_orig $=$ search\&_S o r t $=\mathrm{d} \& \mathrm{v}$ i e $\mathrm{w}=\mathrm{c} \& \quad$ a c c $\mathrm{t}=\mathrm{C} 0000037899$ \&__ version $=1$ \&_u r l Vers i o n $=0$ \&_u s erid=687358\&md5=4a65c7019fbb5efd92531438a6d47a4c. Doi: 10.1016/S0308-8146(03)00159-6.

RAVICHANDRAN, R.; PARTHIBAN R. The impact of mechanization of tea harvesting on the quality of south indian CTC teas. Food Chemistry, London, v.63, n.1, p.61-64, 1998. Disponível em: http://www.sciencedirect.com/ science?_ob=ArticleURL\&_udi=B6T6R-3TPDSC8-

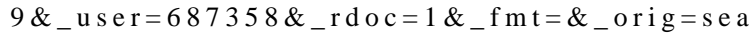
r c h \&_s or t $=$ d \& vi e w = c \&_a c c t = C 000003 $7899 \&$ \& vers i o n $=1 \&_{-}$u r l Vers i o $n=0$ \&_userid=687358\&md5=3db82afa708949197bf60e452db3c41d. Doi: 10.1016/S0308-8146(97)00219-7.

ROSEN, J.F. Effects of low levels of lead exposure. Science, Washington, v.256, n.5055, p.294, 1992.

RUAN, J. et al. Effect of root zone $\mathrm{pH}$ and form and concentration of nitrogen on accumulation of quality-related components in green tea. Journal of the Science of Food and Agriculture, London, v.87, n.8, p.1505-1516, 2007. Disponível em: http://www3.interscience.wiley.com/journal/ 114229333/abstract. Doi: 10.1002/jsfa.2875.

SANDERSON, G.W. et al. Contribution of polyphenolic compounds to taste of tea. American Chemical Society Symposium Series, Washington, v.26, p.14-46, 1976.

SCHARBERT, S. et al. Identification of the astringent taste compounds in black tea infusions by combining instrumental analysis and human bioresponse. Journal of Agricultural and Food Chemistry, Easton, v.52, n.11, p.3498-3508, 2004. Disponível em: http://pubs.acs.org/doi/abs/10.1021/jf049802u. Doi: $10.1021 /$ jf049802u.

SCHÜLLER, H.M. et al. Neuroendocrine lung carcinogenesis in hamsters is inhibited by green tea or theophylline while the development of adenocarcinomas is promoted: implications for chemoprevention in smokers. Lung Cancer, Amsterdam, v.45, n.1, p.11-18, 2004. Disponível em: http://www.sciencedirect.com/ science?_ob=ArticleURL\&_udi=B6T9C-4BP3SDF- 
$1 \&$ \&_user $=687358 \&$ \&doc $=1 \&$ \& $\mathrm{mt}=\&$ \&_orig $=$ search $\&$ _s or t $=$ d \& vi e w = c \&_a c c t $=$ C $000037899 \&$ _ ve r s i o n $=1$ \& _ u r l V e r s i o n $=0$ \& _ u serid $=687358 \&$ md $5=e 308614$ cabc32cd37f0608635b43da96. Doi: 10.1016/j.lungcan.2003.12.007.

SEUREI, P. Tea improvement in Kenya: a review. Tea, Kericho, v.17, p.76-81, 1996.

SHIOKAZI, T. et al. Effects of tea extract, catechins and caffeine against type I allergic reaction, Yakuzaku Zasshi, Tokyo,117, p. 448-454, 1997.

SHU, W.S. et al. Fluoride and aluminium concentrations of tea products from Sichuan Province, PR China. Chemosphere, Oxford, v.52, n.9, p.1475-1482, 2003. Disponível em: http:// www.sciencedirect.com/science?_ob=ArticleURL\&_udi=B6V7448WB27G-3\&_user $=687358 \&$ \&doc $=1 \&$ \& fmt $=$ \&_orig $=$ sea r c h \&_s ort $=$ d \& vi e w $=$ c \&_a c c t $=$ C 0000378 $99 \&$ \&_version $=1 \&$ _ ur l Version $=0 \&$ _ us e ri $\mathrm{d}=687358 \& \mathrm{md} 5=\mathrm{e} 12 \mathrm{ed} 6300 \mathrm{a} 23248 \mathrm{a} 924 \mathrm{c} 201171 \mathrm{e} 6945$. Doi: 10.1016/S0045-6535(03)00485-5.

TAKAHASHI, S. et al. Establishment of a multi-organ carcinogenesis bioassay using rats treated with a combination of five different carcinogens. Journal of Toxicologic Pathology, Tokyo, v.5, v.151-156, 1992.

TAKEO, T. Green tea and semi-fermented teas. In: WILSON, K.C.; CLIFFORD, M.N. (Eds.) Tea: cultivation to consumption. London: Chapman and Hall, 1992. p.413414 .

TANAKA, T. et al. Production of theasinensins A and D, epigallocatechin gallate dimers of black tea, by oxidationreduction dismutation of dehydrotheasinensin. Tetrahedron, Oxford, v.59, n.40, p.7939-7947, 2003. Disponível em: http:// www.sciencedirect.com/science?_ob=ArticleURL\&_udi=B6THR49FXK73-2\&_user $=687358 \&$ \&rdoc $=1 \&$ \&mt $=$ \&_orig $=$ search $\&$ _sort $=$ d\&view $=$ c \&_acct $=$ C $000037899 \&$ _version $=1 \&$ _ur l Versio n $=0$ \& _ u s e ri d = $687358 \&$ m d $\overline{5}=8480791620800 \mathrm{eb} 531855 \mathrm{e} 63 \mathrm{~b} 495 \mathrm{~d} 32 \mathrm{a}$. Doi: 10.1016/ j.tet.2003.08.025.

TANAKA, T.; KOUNO, I. Oxidation of tea catechins: chemical structures and reaction mechanism. Food Science and Technology Research, Tsukuba, v.9, n.2, p.128-133, 2003. Disponível em: http://www.jstage.jst.go.jp/article/fstr/9/2/9_128/ _article. Doi: 10.3136/fstr.9.128.

THOMAS J. et al. Influence of age after pruning on the levels of flavanols and other bioconstituents in tea (Camellia sinensis). Journal of the Science of Food and Agriculture, London, v.85, n.6, p.931-934, 2005.

VEGRO, C.L.; BEMELMANS, P. Proposta de plano de recuperação da teicultura do Vale do Ribeira. Informações Econômicas, São Paulo, v.26, n.6, p.100-104, 1996.
VENKATESAN, S.; GANAPATHY, M.N.K. Impact of nitrogen and potassium fertiliser application on quality of CTC teas. Food Chemistry, London, v.84, n.3, p. 325-328, 2004. Disponível em: http://www.sciencedirect.com/ science?_ob=ArticleURL\&_udi=B6T6R-491C15S$5 \&$ \&user $=687358 \&$ \&doc $=1 \&$ \&fmt $=\&$ \&_orig $=$ search $\&$ _sort $=$ d \& vi e w = c \&_a c c t $=$ C $000037899 \&$ _ versi o $\mathrm{n}=1 \&$ _ u r l V e r s i o $\mathrm{n}=0 \&$ _ u s e r i d $=6$ 87358\&md5=049c987d62661101ec20496bd6ca557d. Doi: 10.1016/S0308-8146(03)00215-2.

VITORELLO, V.A. et al. Recent advances in aluminum toxicity and resistance in higher plants. Brazilian Journal of Plant Physiology, Londrina, v.17, n.1, p.129-143, 2005.

WACHIRA, F.N. Breeding and clonal selection in relation to black tea quality: a review. Tea, Kericho, 15, p.56-66, 1994.

WALTON, J. et al. Uptake of trace amounts of aluminium into the brain from drinking water. Neuro Toxicology, Little Rock, v.16, p.187-190, 1995.

WANG, Y. et al. Discussion on the chemical standards on quality of Chinese roasted green tea. Journal of Tea Science, Hangzhou, v.8, p.13-20, 1988.

WANG, H. et al. Tea flavonoids: their functions, utilisation and analysis. Trends in Food Science and Technology, Amsterdam, v.11, n.4-5, p.152-160, 2000. Disponível em: http:// www.sciencedirect.com/science?_ob=ArticleURL\&_udi=B6VHY41Y85ND-5\&_user $=687358 \&$ _rdoc $=18$ _fmt $=$ \&_orig $=$ search\&_so $\mathrm{rt}=\mathrm{d} \& \mathrm{v}$ i e $\mathrm{w}=\mathrm{c} \&$ _ a c c t $=$ C $000037899 \&$ _ versio n $=1 \&$ _ u r l Versio $n=0 \&$ _ u s e r id=687358\&md5=662021bbd17ada8e564aee2aceb5de76. Doi: 10.1016/S0924-2244(00)00061-3.

WANG, L.F. et al. The compounds contributing to the greenness of green tea. Journal of Food Science, Chicago, v.69, n.8, p.301-305, 2004.

XIE, M.Y. et al. Multielement analysis of Chinese tea (Camellia sinensis) by total-reflection X-ray fluorescence. Zeitschrift fur Lebensmittel-Untersuchung und Forschung . A, Berlin, v.207, p.31, 1998. Disponível em: http://www.springerlink.com/ content/ttwkhwxq2kch091j/. Doi: 10.1007/s002170050291.

YANG, S.C.; WANG, Z. Tea and cancer. Journal of the National Cancer Institute, Bethesda, v.85, n.13, p.10381049, 1993.

ZHU, Y-X. et al. A review of recent studies in China on the possible beneficial health effects of tea. International Journal of Food Science and Technology, Oxford, v.41, n.4, p.333-340, 2006. Disponível em: http:// www3.interscience.wiley.com/journal/118573310/abstract. Doi: 10.1111/j.1365-2621.2005.01076.x. 